\title{
Injection in light beam induced current systems: An analytical model
}

\author{
Gabriel Micard*, Giso Hahn, and Barbara Terheiden \\ Department of Physics, University of Konstanz, 78457 Konstanz, Germany
}

Keywords carrier concentration, LBIC, light beam induced current, $\mathrm{p}-\mathrm{n}$ junction, photovoltaics, solar cells

*Corresponding author: e-mail gabriel.micard@uni-konstanz.de, Phone: +49 753188 2060, Fax: +49 7531883685

In LBIC systems, the evaluation of the injection level is necessary when operating in low injection for defect recombination studies or in defined standard illumination conditions (one or several suns for concentrator applications) for quantum efficiency evaluation. We demonstrate in this contribution that evaluating the laser beam induced injection based on uniform illumination condition can lead to several decades of error because of the lateral carrier diffusion. Based on a parallel beam approximation, we propose here an analytical model to evaluate the maximum of injection of a laser with its parametrization valid for most LBIC system settings and material quality. State of the art high resolution LBIC (HR-LBIC) systems have so sharply focused laser beams that the beam divergence cannot be neglected anymore in the injection calculation. Although providing a quantitative criterion to determine whether the beam divergence can be neglected, we provide a more advanced model for describing the injection of the laser that includes beam divergence.
1 Introduction In solar cell research, light beam induced current (LBIC) has become a standard scanning technique to investigate local defects leading to solar cell efficiency losses [1-3]. LBIC is used in first intention to show qualitatively the spatially resolved defect distribution in the solar cell, however, to reveal their quantitative impact on the performance of the solar cells (local internal quantum efficiency, IQE) in operating conditions (one or several suns) the injection in the bulk around the laser spot must be accurately tuned.

One should ensure that the injection distribution around the laser spot is identical (at least similar) to the one induced by uniform illumination used in operating conditions.

While this can be achieved by using bias light [4] illuminating uniformly around the lasers spot, this method induces a large dc current $[4,5]$ that is difficult to cope with and might be additionally technically difficult to implement in high resolution LBIC (HR-LBIC) systems because of the small focal length of the laser focusing lens [6, 7].

An alternative method using the laser beam as an illumination bias, while being not ideal because of the nonuniformity of the laser illumination, could be, however, relevant providing that the maximum injection induced by the laser beam matches the one induced by the reference uniform illumination.
The issue of estimating injection maxima in both cases arises also for LBIC systems using lamp and monochromator as light source [8] as well as solar LBIC systems [9], because of focusing the light sharply. In these cases, focusing the light beam creates a Gaussian distribution of light intensity that might hinder the requirement of spatial uniformity of the light source. This situation leads to the same non-ideal situation than with the lasers with, however, the same requirements in terms of injection distribution.

It is then tempting to tune the laser (or light) power so that its maximum power density (at the center of the beam) equals the spatially uniform power density required by the standard operating condition of the solar cell. This implicitly assumes that the injection in both cases will be similar.

However, the injection reached in case of illumination by laser might be highly overestimated because the carriers generated by laser illumination can also diffuse laterally due to spatial non-uniformity of the laser illumination.

We demonstrate that such overestimation remains acceptable only if the laser spot size is much larger than the diffusion length. We propose an analytical model for cases where such assumption is not reasonable.

Most commonly used LBIC setups [2, 4, 9-11] focus the lasers to minimal radii of $6 \mu \mathrm{m}$ for which the beam divergence can be in general neglected. We propose for this 
situation a parallel beam model in which the injection in the center of the laser beam is computed from the beam radius, light penetration depth, and diffusion length. To determine quickly the adjustments in laser power or focus necessary for the most common focusing conditions and material quality, we provide a simple parametrization in terms of two independent variables that is fully consistent with the uniform illumination model.

However, state of the art HR-LBIC systems use a beam expander [12] before focusing allowing radii down to $0.6 \mu \mathrm{m}$. In this case, a more advanced model including the effect of beam divergence is provided. This model, however, cannot be easily parameterized due to its enhanced complexity.

Although a rigorous calculation of the injection is beyond the scope of this article, we aim here at providing expressions (and parametrization when available) to evaluate the effect of such lateral diffusion on the injection with respect to a uniform illumination valid on the full usual range of applicability of LBIC systems.

We finally mention that the maximum injection of the laser beam is also required in LBIC systems used to extract intrinsic properties of defects. In this case, laser intensity should be low enough to avoid saturating minority carriers [9] which allows considering the recombination properties to be independent of injection. A careful evaluation of the maximum of injection becomes as important for such setups as it is for electron beam induced current (EBIC) systems used for these investigations [13]. However, evaluating the amplitude of the injection is not trivial because of the two-dimensional nature of the generation function in cylindrical coordinates which cannot be approximated as a sphere like assumed for EBIC [13].

\section{Problem description}

\subsection{Shallow junction in short-circuit condition}

The minority carrier spatial distribution $p\left(r_{\mathrm{G}}\right)$ in the bulk of a solar cell is obtained by solving explicitly the steady state diffusion equation

$$
\Delta p\left(r_{\mathrm{G}}\right)-\Lambda^{2} p\left(r_{\mathrm{G}}\right)=-g\left(r_{\mathrm{G}}\right) / D
$$

with $\Delta$ the Laplacian operator, $r_{\mathrm{G}}$ the generalized coordinate, $\Lambda=1 / L_{\text {diff }}$ with $L_{\text {diff }}$ the minority carrier diffusion length in the semiconductor that is assumed homogeneous over the whole bulk volume, $g\left(r_{\mathrm{G}}\right)$ the volume generation function of the illumination source and $D$ the minority carrier diffusion coefficient.

To solve Eq. (1) we make the assumption of infinite thickness, implying that we solve on the half space $z>0$ with the junction boundary condition (BC) at $z=0$. Strictly speaking, this assumption is justified only when the light penetration depth $L_{\alpha}=\alpha^{-1}$ ( $\alpha$ being the optical absorption coefficient) and $L_{\text {diff }}$ are smaller than the wafer thickness $t$. The influence of the back surface on the injection close to the front surface is, however, minimal because $g\left(r_{\mathrm{g}}\right)$ has its maximum at the front surface.
Because the problem and the generation source (laser beam or uniform illumination) has a cylindrical symmetry, the problem reduces to two dimensions in cylindrical coordinates (radius $r$ and depth $z$ ) leading to this other expression of Eq. (1).

$$
\frac{\partial^{2} p}{\partial r^{2}}+\frac{1}{r} \frac{\partial p}{\partial r}+\frac{\partial^{2} p}{\partial z^{2}}-\Lambda^{2} p=-g(r, z) / D
$$

In an LBIC measurement, the junction operates in short circuit condition implying $p_{z=0}=p_{0}$ where $p_{0}$ is the equilibrium density of minority carriers in the wafer. However, $p_{0}$ is always very small in comparison to any injection level induced by the illumination source and therefore the simpler $\mathrm{BC} p_{z=0}=0$ is used.

Strictly speaking, this BC applies at the edge of the space charge region (SCR) and not at the semiconductor surface. Although it is customary to assume the junction and space charge thickness $z_{\mathrm{j}}$ to be very small with respect to $L_{\alpha}$ which allows neglecting it, this assumption breaks down for sharply focused laser LBIC system with relatively short wavelength [12].

For completeness, $z_{\mathrm{j}}$ will be integrated into all further developed models by shifting the $z$-axis of the generation function by $z_{\mathrm{j}}$.

2.2 Generation function In all generation expressions developed in this section, the front surface is assumed to be perfectly flat corresponding to the case of a polished surface. In the case of a real solar cell-textured surface, considering a local laser illumination, the generation function will depend explicitly on the position of the laser beam on the surface which, on a random surface geometry, cannot be integrated in such analytical theory. One may consider that, by increasing the light scattering at the front surface, the maximum of generation function (but of course not the total number of generated electron hole pair in the volume) and thus the maximum of injection will be reduced with respect to a flat surface. Therefore, the model presented in this article allows for the calculation of an upper bound to the maximum injection reached when considering a textured surface.

For spatially uniform illumination, the intensity decay of light intensity in an absorbing media is simply described by the Lambert-Beer law taking into account the reflection coefficient at the semiconductor surface $R_{\mathrm{f}}$.

$$
I_{\text {uni }}(z)=\Phi_{\text {uni }}\left(1-R_{\mathrm{f}}\right) \cdot \exp (-\alpha z)
$$

with $\Phi_{\text {uni }}$ the uniform illumination power density and $\alpha$ the optical absorption coefficient.

Taking its derivative in $z$ direction allows then to get the volumic rate of energy absorption which, normalized by the photon energy $E_{\mathrm{ph}}$, gives the generation function $g_{\text {uni }}$.

$$
g_{\text {uni }}\left(r_{\mathrm{g}}\right)=\frac{\eta \alpha}{E_{\mathrm{ph}}} I_{\text {uni }}\left(r_{\mathrm{g}}\right)
$$


with $\eta$ the conversion ratio between photon and electronhole pairs (usually $\eta=1$ is assumed) and $E_{\mathrm{ph}}=h_{\mathrm{p}} \cdot c / \lambda_{0}$ in which $h_{\mathrm{p}}$ is the Planck's constant, $c$ the speed of light in vacuum, and $\lambda_{0}$ is the photon wavelength in vacuum.

The Lambert-Beer law, however, applies only to a planar electromagnetic wave that is not the case of a focused Gaussian laser beam. We, therefore, consider more generally the expression of volumic density of optical power absorbed during the propagation of an arbitrary electromagnetic wave in an absorbing media [14].

$$
W=\frac{1}{2} \sigma|E|^{2}=\frac{1}{2} \frac{2 \pi \cdot \mathrm{c} \cdot \varepsilon_{0} \operatorname{Im}\left(\varepsilon_{\mathrm{r}}\right)}{\lambda_{0} \mu_{\mathrm{r}}}|E|^{2}
$$

where $E$ is the electric field of the radiation and $\sigma$ is the conductivity of the media linked to its complex relative permittivity $\varepsilon_{\mathrm{r}}$ and its relative permeability $\mu_{\mathrm{r}}$ whereas the latter equals 1 for a non-magnetic material.

The complex refractive index $\tilde{n}$ of a material is defined as

$$
\tilde{n}=n+i k,
$$

with $n$ and $k$ its real and imaginary part.

The relative permittivity of this material is linked to its complex refractive index by the relation $\tilde{n}^{2}=\varepsilon_{\mathrm{r}}$ leading to express $\varepsilon_{\mathrm{r}}$ as

$$
\varepsilon_{\mathrm{r}}=n^{2}-k^{2}+2 i n k .
$$

Assuming the Gaussian wave to be locally planar (paraxial approximation) one can define its intensity as [15]

$$
I_{\text {Gaus }}=\frac{c \varepsilon_{0} n}{2}|E|^{2} \text {. }
$$

Introducing the imaginary part of Eq. (7) into Eq. (5) and identifying $I_{\text {Gaus }}$ leads to

$$
W=I_{\text {Gaus }} \frac{4 \pi k}{\lambda_{0}} .
$$

Normalizing Eq. (9) by $E_{\mathrm{ph}}$ and $\eta$ like for Eq. (4) and recognizing the optical absorption coefficient $\alpha$ defined by $4 \pi k / \lambda_{0}$ leads to

$$
g_{\text {Gaus }}\left(r_{\mathrm{g}}\right)=\frac{\eta \alpha}{E_{\mathrm{ph}}} I_{\text {Gaus }}\left(\mathrm{r}_{\mathrm{g}}\right),
$$

while identical to the expression derived for the uniform illumination case (Eq. (4)) this result is, however, more general as it requires only paraxial waves and thus could be used for spherical waves far enough from the source.

In general, the intensity of a Gaussian beam focused at the surface (with a reflection coefficient $R_{\mathrm{f}}$ ) of a semiinfinite uniform absorbing media can be described in cylindrical coordinates as [16]
$I(r, z)=\Phi_{0, \text { laser }}\left(1-R_{\mathrm{f}}\right) \frac{\exp (-\alpha z)}{d(z)} \exp \left(-\frac{2 c(z)}{\sigma_{0}^{2} d(z)} r^{2}\right)$,

with $\sigma_{0}$ the beam waist radius at normalized intensity $1 / e^{2}$ and the maximum impinging laser intensity $\Phi_{0, \text { laser }}=2 P_{\text {laser }} /\left(\sigma_{0}^{2} \pi\right)$ in which $P_{\text {laser }}$ is the total power of the laser. The distortion of the curvature due to absorption $c(z)$ and the broadening of the laser beam $d(z)$ are expressed as

$$
\begin{aligned}
& c(z)=1+\frac{k}{|\tilde{n}|}\left(\frac{z}{z_{\mathrm{r}}}\right), \\
& d(z)=1+\frac{2 k}{|\tilde{n}|}\left(\frac{z}{z_{\mathrm{r}}}\right)+\left(\frac{z}{z_{\mathrm{r}}}\right)^{2}, \\
& z_{\mathrm{r}}=\pi|\tilde{n}| \sigma_{0}^{2} / \lambda_{0},
\end{aligned}
$$

with $z_{\mathrm{r}}$ as the Rayleigh range of the beam, $n$ and $k$ the real and imaginary part of the complex refractive index $\tilde{n}$.

We want to point out that to our knowledge, the injection induced by a laser beam cannot be computed analytically from this generation function without approximation.

The strongest approximation (parallel beam approximation) is obtained by considering $c(z)=1$ and $d(z)=1$ implying $z \ll z_{\mathrm{r}}$ and $z \ll z_{\mathrm{r}} \cdot|\tilde{n}| /(2 k)$.

As we assume infinite thickness, one directly sees that these conditions will not be respected for very large $z$. One could note, however, that in this case the beam might be already so much absorbed that inaccuracy in its description will not affect significantly the generation function.

We, therefore, consider that the influence of absorption on curvature could be neglected if one fourth of $|\tilde{n}| \cdot z_{\mathrm{r}} /(2 k)$ is larger than four times the penetration depth. Using the definition of $\alpha=4 \pi k / \lambda_{0}$ the constraint $|\tilde{n}| \cdot z_{\mathrm{r}} /(8 k)>4 / \alpha$ leads to the following condition for $\sigma_{0}$ :

$$
\sigma_{0}>2^{3 / 2} \sigma_{0 \min } /|\tilde{n}|
$$

where $\sigma_{0 \min }=\lambda_{0} / \pi$ is the minimum radius reachable by focusing in the vacuum (or air) [17]. We can then observe that in the case of crystalline silicon and many semiconductor materials $2^{3 / 2} /|\tilde{n}|<1$ or $\tilde{n}>2.818$ is always fulfilled in the LBIC relevant wavelength range. Therefore, the critical radius below which this effect has to be considered has to be smaller than the minimum radius imposed by diffraction. This condition is, therefore, always guaranteed and $c(z)=1$ will be safely taken in the following of this article.

While this approximation was shown to be very good for an amplifying media [18], a similar demonstration was not performed, to our knowledge, for an absorbing media.

Considering the aforementioned condition on $c(z)$ to be fulfilled (allowing also to neglect the linear term in the 
expression of $d(z))$, a similar demonstration for the quadratic term in divergence of the beam $d(z)$ gives the constraint $z_{\mathrm{r}} / 4>4 / \alpha$ that leads to the following condition for $\sigma_{0}$ :

$$
\sigma_{0}>2 \sigma_{0 \min } / \sqrt{k|\tilde{n}|}
$$

when this condition is also fulfilled, $d(z)=1$ can be taken and the beam can be considered as parallel. Figure 1 shows this condition for crystalline silicon as a function of laser wavelength. One can then observe that though only very small radii do not fulfill this condition some experimental setups do focus so sharply [12] at high wavelength. Thus, $d(z)=1+\left(z / z_{\mathrm{r}}\right)^{2}$ if the beam is treated as diverging or $d(z)=1$ if treated as parallel.

The next section describes the case of a spatially uniform illumination as a point of reference, followed in the Section 4 and 5 by the development of the model for a parallel laser beam and diverging beam, respectively.

3 Spatially uniform illumination (bias light) In order to compare to the laser illumination, we first derive an analytic expression for the injection maximum in the case of uniform illumination. The generation function for this problem is obtained straightforwardly from Eqs. (3) and (4) by shifting the $z$-axis by $z_{\mathrm{j}}$ (see end of the Section 2.1)

$$
g(z)=g_{0, \text { uni }} \mathrm{e}^{-\alpha z_{j}} \cdot \exp (-\alpha z)
$$

with

$$
g_{0, \text { uni }}=\Phi_{\text {uni }} \times E_{\mathrm{ph}} \times\left(1-R_{\mathrm{f}}\right) \times \eta \alpha .
$$

We see that in this case, integrating $z_{\mathrm{j}}$ in the generation function leads just to a scaling factor or an effective reduction of the light illumination. Because $g$ depends only on $z$, the injection is only a function of $z$ and the partial

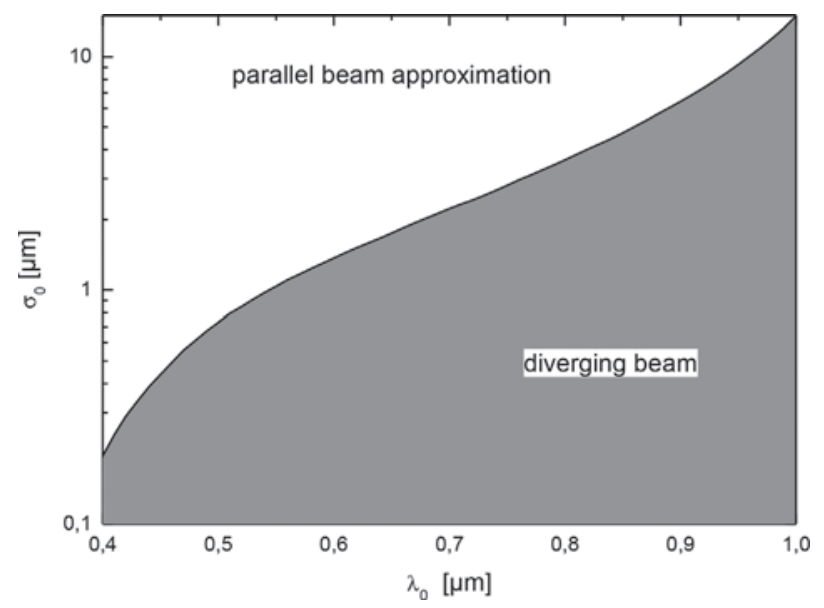

Figure 1 Range of validity for crystalline silicon of injection theories using a parallel beam approximation versus the one requiring to take the divergence into account. differential equation (PDE) of Eq. (2) reduces to the following ordinary differential equation (ODE) in $z$.

$$
\frac{\mathrm{d}^{2} p}{\mathrm{~d} z^{2}}-\Lambda^{2} p=-\left(g_{0, \text { uni }} \mathrm{e}^{-\alpha z_{j}} / D\right) \exp (-\alpha z) .
$$

The solution obtained by standard methods is

$$
p(z)=\frac{g_{0, \text { uni }} \mathrm{e}^{-\alpha z_{j}}}{D} \cdot \frac{\exp (-\alpha z)-\exp (-\Lambda z)}{\Lambda^{2}-\alpha^{2}} .
$$

Taking the derivative of Eq. (20) and equating it to 0 leads to the expression for the location of the injection maximum

$$
z_{\max }=\frac{\ln (\alpha / \Lambda)}{\alpha-\Lambda}=L_{\mathrm{diff}} \frac{\ln \left(\alpha L_{\mathrm{diff}}\right)}{\alpha L_{\mathrm{diff}}-1} .
$$

Then replacing this location in Eq. (20) gives the value of maximum injection,

$$
p_{\max }=p_{00, \text { uni }} \mathrm{e}^{-\alpha z_{j}} \frac{X^{\frac{X}{1-X}}}{X+1},
$$

with $X=\alpha \cdot L_{\mathrm{diff}}$ :

$$
p_{00, \text { uni }}=\frac{g_{0, \text { uni }} L_{\mathrm{diff}}^{2}}{D}=\frac{\eta\left(1-R_{\mathrm{f}}\right)}{E_{p h}} \cdot \frac{\Phi_{0 \text { uni }} \alpha}{D} L_{\mathrm{diff}^{2}}^{2} .
$$

Though the function in $X$ in Eq. (22) is almost fully analytical (except for $X=1$ where its limit gives the value $e^{-1 / 2}$ ) an accurate and simpler parametrization can be obtained as

$$
p(0, z)=p_{00, \text { uni }} \mathrm{e}^{-\alpha z_{j}} \cdot\left(1+A_{1} X+A_{2} X^{2}\right)^{-1},
$$

with $A_{1}=A_{1 \mathrm{u}}=3.4523$ and $A_{2}=A_{2 \mathrm{u}}=1.0019$. This parameterization will serve as a basis to the parametrization of the laser injection model

4 Model for a parallel beam Figure 2 shows a typical volume generation function as well as the associated carrier density distribution. One can observe the lateral carrier density broadening due to carrier diffusion as well as the 0 carrier density at the surface imposed by the junction in short circuit.

Equations leading to the solution of this problem were presented by Marek [19] by transforming the PDE of Eq. (2) in an ODE in $z$ making the use of the Hankel transform [20]. In the case of a parallel beam, an alternative derivation by transforming the PDE of Eq. (2) in an ODE in $r$ is presented here. The solution following Marek's path is presented after.

4.1 Solutions at the center of the beam Using Green's function method to solve this PDE problem, the Green's function is solution of Eq. (2) with a point source at 


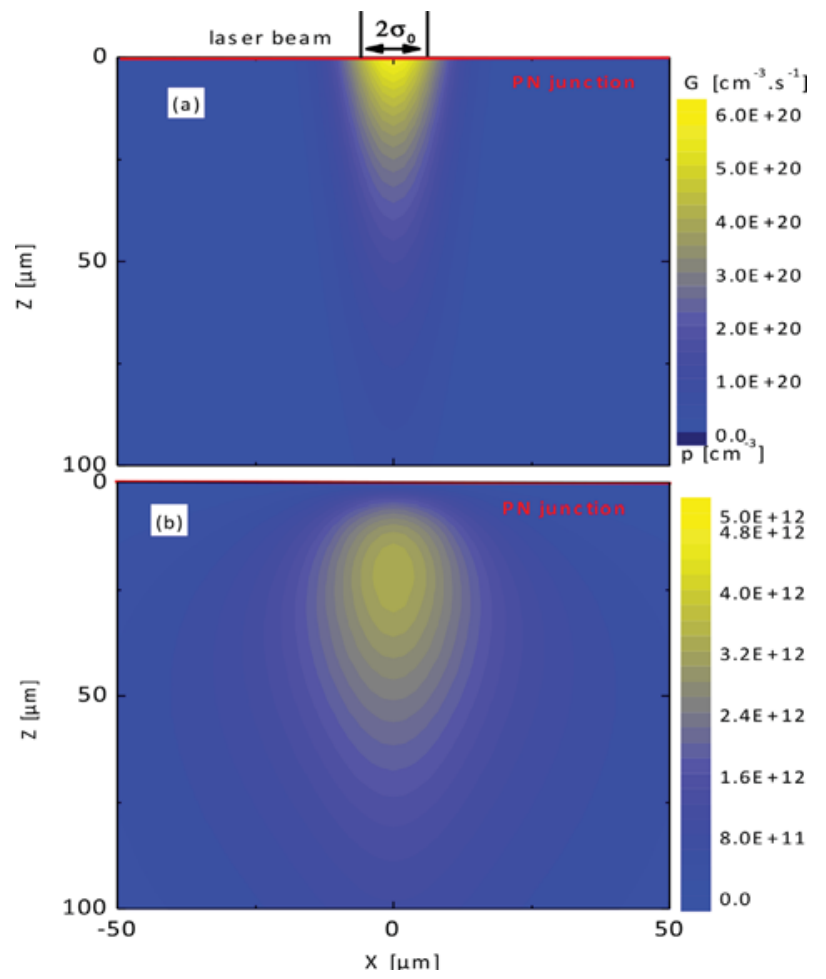

Figure 2 (a) Volume generation function $g$ for a laser beam of wavelength $\lambda=910 \mathrm{~nm}\left(\alpha=27 \mathrm{~mm}^{-1}\right)$, a beam radius of $\sigma_{0}=10$ $\mu \mathrm{m}$, and a power $P_{\text {laser }}=1.5 \mu \mathrm{W}$ assuming no front reflection. (b) Cross-section of the carrier density distribution in a n-type bulk of diffusion length $L_{\text {diff }}=100 \mu \mathrm{m}$ assuming a hole diffusion constant $D=12 \mathrm{~cm}^{2} \mathrm{~s}^{-1}$ when the shallow junction is in short circuit.

$$
\begin{aligned}
& r=r_{\mathrm{p}} \text { and } z=z_{\mathrm{p}} \\
& \frac{\partial^{2} G}{\partial r^{2}}+\frac{1}{r} \frac{\partial G}{\partial r}+\frac{\partial^{2} G}{\partial z^{2}}-\Lambda^{2} G=\delta\left(r-r_{\mathrm{p}}\right) \delta\left(z-z_{\mathrm{p}}\right) .
\end{aligned}
$$

The PDE of Eq. (25) can be transformed into an ODE in $r$ making use of the sine Fourier transform [21] that obeys this surface BC

$$
G\left(r, r_{\mathrm{p}}, z, z_{\mathrm{p}}\right)=\int_{0}^{\infty} b\left(r, r_{\mathrm{p}}, k, z_{\mathrm{p}}\right) \sin (z k) \mathrm{d} k
$$

leading to

$$
\frac{\partial^{2} b}{\partial r^{2}}+\frac{1}{r} \frac{\partial b}{\partial r}-\mu^{2} b=A\left(z_{\mathrm{p}}\right) \delta\left(r-r_{\mathrm{p}}\right)
$$

in which $A\left(z_{\mathrm{p}}\right)=(2 / \pi) \sin \left(z_{\mathrm{p}} k\right)$ and $\mu=\left(\Lambda^{2}+k^{2}\right)^{1 / 2}$. This ODE can be rearranged in a modified Bessel equation, and its linearly independent solutions are the modified Bessel functions of order $0, K_{0}(\mu r)$, and $I_{0}(\mu r)$ (Section 9.6 in Ref. [22]). The particular solution is a piece-wise function that can be derived using the fundamental theorem of Green's function as described in detail in Ref. [23] leading to

$$
\begin{aligned}
& b^{-}\left(r<r_{\mathrm{p}}\right)=-A\left(z_{\mathrm{p}}\right) r_{\mathrm{p}} K_{0}\left(\mu r_{\mathrm{p}}\right) I_{0}(\mu r) \\
& b^{+}\left(r>r_{\mathrm{p}}\right)=-A\left(z_{\mathrm{p}}\right) r_{\mathrm{p}} I_{0}\left(\mu r_{\mathrm{p}}\right) K_{0}(\mu r) .
\end{aligned}
$$

From this expression, the full carrier distribution would involve a double integral that cannot be brought to a closed form. If, however, one has the objective only to get a closed form expression for the maximum of injection, it can be stated that because the source has a cylindrical symmetry with a maximum generation at the center $(r=0)$, the maximum of injection should also be in the center and we can therefore restrict our solution to the center of the laser beam (Fig. 2). In such case, we are only interested in the $b^{-}$solution of Eq. (28), because the point source has necessarily a location $r=0<r_{\mathrm{p}}$.

Then using the property that $I_{0}(0)=1$ in the $b^{-}$ expression of Eq. (28), Eq. (26) leads to

$$
G\left(0, r_{\mathrm{p}}, z, z_{\mathrm{p}}\right)=-\int_{0}^{\infty} A\left(z_{\mathrm{p}}\right) r_{\mathrm{p}} K_{0}\left(\mu r_{\mathrm{p}}\right) \sin (z k) \mathrm{d} k .
$$

The final solution for the PDE problem can be expressed as a convolution product over the volume of definition

$p(0, z)=\int_{0}^{\infty} \int_{0}^{\infty} G\left(0, r_{\mathrm{p}}, z, z_{\mathrm{p}}\right)\left(-g\left(r_{\mathrm{p}}, z_{\mathrm{p}}\right) / \mathrm{D}\right) \mathrm{d} r_{\mathrm{p}} \mathrm{d} z_{\mathrm{p}}$.

The generation function for this problem is obtained straightforwardly from Eqs. (9) and (10) with $d(z)=1$ and shifting the $z$-axis by $z_{\mathrm{j}}$ (see end of the Section 2.1)

$$
\begin{aligned}
g(r, z)= & g_{0, \text { laser }} \cdot \mathrm{e}^{-\alpha z_{j}} \exp (-\alpha z) \\
& \times \exp \left(-2 r^{2} / \sigma_{0}^{2}\right),
\end{aligned}
$$

with

$$
g_{0, \text { laser }}=\frac{\eta \alpha}{E_{\mathrm{ph}}} \Phi_{0, \text { laser }}\left(1-R_{\mathrm{f}}\right)
$$

Identically to the uniform illumination model, integrating $z_{\mathrm{j}}$ leads just to a scaling factor or an effective reduction of the light illumination. Making use of Eqs. (29) and (31) and assuming that the order of the integral can be permuted, Eq. (30) could be rewritten after reorganization as

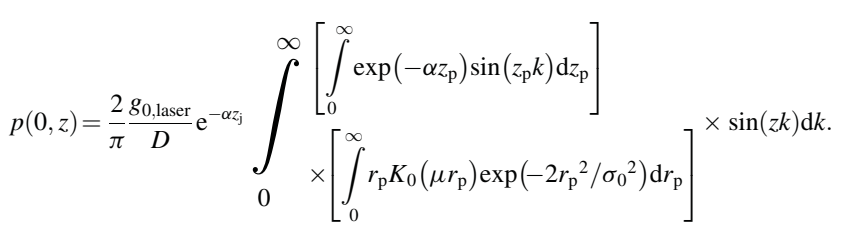

While the integral in $z_{\mathrm{p}}$ has a simple analytical solution, the integral in $r_{\mathrm{p}}$ is more elaborated. The analytic expression of a similar integral can be found in Section 6.631 Eq. (3) of 
Ref. [24]. However, this solution involves a Whittaker function with three parameters and thus requires additional calculations to be simplified for this particular case making use of Eq. (13.1.33) and Eq. (13.6.30) in [22]. After integral solving, replacing $\mu$ by its expression and reorganizing Eq. (33) leads to

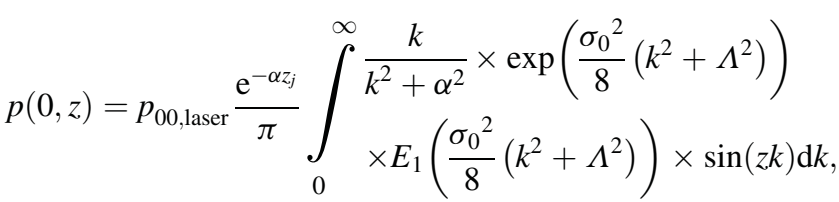

where $E_{1}(x)$ is the exponential integral function (see the Section 5 in Ref. [16] for details). Realizing that the integral is dimensionless, it implies that the prefactor $p_{00, \text { laser }}$ is a carrier density that could be expressed as (while replacing $g_{0, \text { laser by its value) }}$

$$
p_{00, \text { laser }}=\frac{\sigma_{0}^{2}}{4} \frac{g_{0, \text { laser }}}{D}=\frac{\eta\left(1-R_{\mathrm{f}}\right)}{E_{\mathrm{ph}}} \frac{\Phi_{0, \text { laser }} \alpha}{D} \frac{\sigma_{0}^{2}}{4} .
$$

Replacing $\Phi_{0, \text { laser }}$ by its expression we see that $p_{00 \text {,laser }}$ is independent of the beam waist radius $\sigma_{0}$ whose contribution is exclusively in the integral.

Following Marek's path, we assume $\sigma(z)=\sigma_{0}$ in Eq. (4) in Ref. [19] and observe that the generation distribution used by Marek is identical to ours to some factor. Therefore, restricting the solution to the center of the laser beam $(r=0)$ and using the fact that $J_{0}(0)=1$ with $J_{0}$ the Bessel function of the first kind of order 0 in Eq. (5) in Ref. [19], this equation could be reformulated for our problem as

$$
\begin{aligned}
p(0, z)= & p_{00, \text { laser }} \cdot \mathrm{e}^{-\alpha z_{\mathrm{j}}} \\
& \times \int_{0}^{\infty} \frac{k}{2 \mu} \mathrm{e}^{-k^{2} \sigma_{0}^{2} / 8} \int_{0}^{\infty}\left[\mathrm{e}^{-\mu\left|\mathrm{z}-z_{\mathrm{p}}\right|}-\mathrm{e}^{-\mu\left(z+z_{\mathrm{p}}\right)}\right] \mathrm{e}^{-\alpha z_{\mathrm{p}}} \mathrm{d} z_{\mathrm{p}} \mathrm{d} k
\end{aligned}
$$

and the integration over $z_{\mathrm{p}}$ can be performed taking into account the sign of the absolute value leading to the equivalent relation to Eq. (34) as

$p(0, z)=p_{00, \text { laser }} \cdot \mathrm{e}^{-\alpha z_{\mathrm{j}}} \int_{0}^{\infty} \frac{k \cdot \exp \left(-k^{2} \sigma_{0}^{2} / 8\right)}{k^{2}+\Lambda^{2}-\alpha^{2}}\left(\mathrm{e}^{-\alpha z}-\mathrm{e}^{-\mu z}\right) \mathrm{d} k$.

\subsection{Comparison of the model expressions} Because they are derived from the same problem with the same assumptions but different ways, Eqs. (34) and (37) give the same numerical result though a transformation from one to the other has not been found. These two equations can, therefore, bring different aspect of the same solution.
As Eq. (34) is a sine Fourier transform, the integrand (without the sine function) can be interpreted as the spectrum of the $z$ distribution of the injection, leading to possible further analysis using fast Fourier transform (FFT). On the other hand, Eq. (37) presents similarities with Eq. (20) allowing considering the uniform illumination as a limiting case of the parallel beam illumination. This property will be useful for the model parameterization.

One can remark in Fig. 3 that the maximum injection value becomes much more independent of $L_{\text {diff }}$ for small $\sigma_{0}$. This property can be inferred relatively straightforwardly from Eq. (34) observing that the contribution of $L_{\text {diff }}$ in this equation comes only from $\sigma^{2} \Lambda^{2}=\left(\sigma_{0} / L_{\text {diff }}\right)^{2}$ that is very small when $L_{\text {diff }} \gg \sigma_{0}$.

Both equations require numerical evaluation which is easier with Eq. (37) as the integrand is not oscillatory.

4.3 Discussion A general comment about Fig. 3 is that all curves decay toward low $L_{\text {diff }}$ because of the increasing bulk recombination.

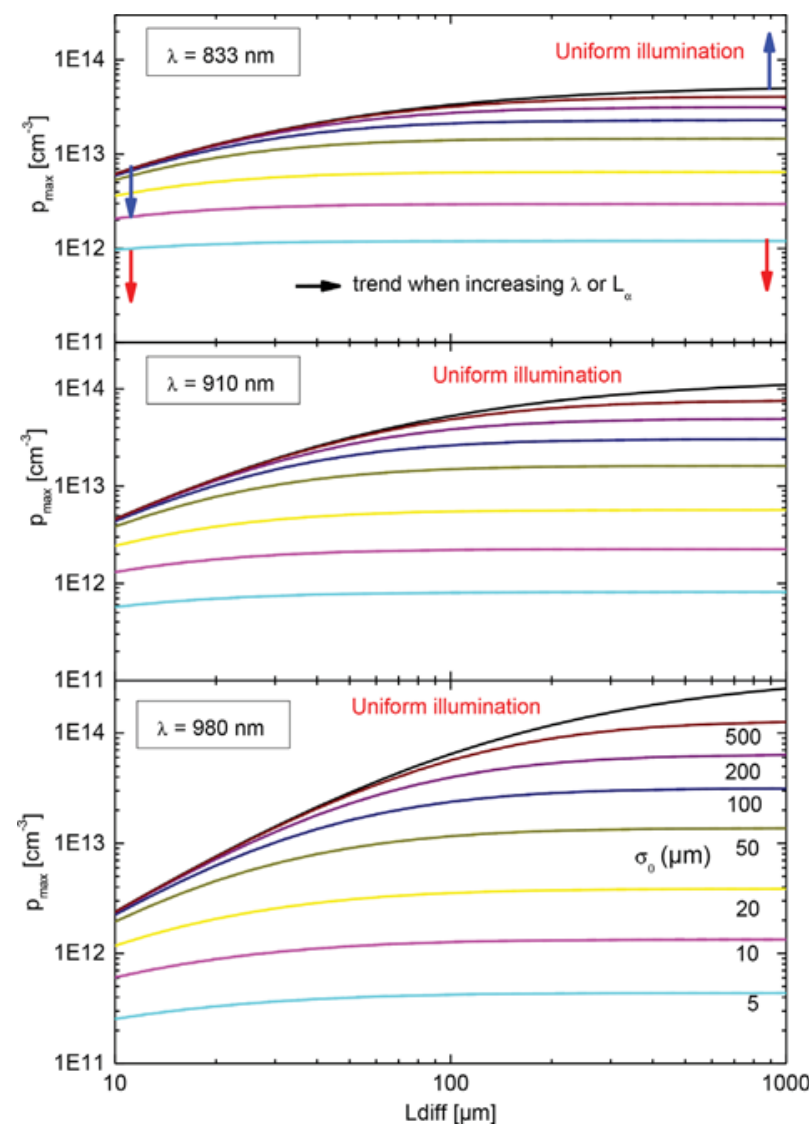

Figure 3 Comparison of the maximum injection induced by laser beam with typical wavelength $\lambda=833,910$, and $980 \mathrm{~nm}(\alpha=64$, 27 , and $9.6 \mathrm{~mm}^{-1}$ ) and the same maximum photon flow as a uniform photon flow of $4.2 \times 10^{17}$ photons s${ }^{-1} \mathrm{~cm}^{-2}$ (corresponding to $\Phi_{\max }=\Phi_{\text {uni }}=0.1 \mathrm{~W} \mathrm{~cm}^{-2}$ at $\lambda=833 \mathrm{~nm}$ ) for various laser beam radii in a wafer of various diffusion length $L_{\text {diff }}$ and a diffusion constant $D=12 \mathrm{~cm}^{2} \mathrm{~s}^{-1}$. 
One can see in Fig. 3 that for large $\sigma_{0} \gg L_{\text {diff }}$ the maximum of injection tends to the maximum reached during uniform illumination. Indeed, the lateral carrier diffusion at the periphery of the beam generation influences only weakly the injection in its center where the diffusion occurs mainly vertically. This statement can be also derived mathematically because the parallel beam model equation (Eq. (34)) tends to the uniform model equation (Eq. (20)) when $\sigma_{0}$ tends to infinity (demonstration in Appendix $\mathrm{M}$ in Ref. [23]).

In the case of uniform illumination, we observe in Fig. 3 that raising photon wavelength and so the penetration depth leads to an increase of injection for high $L_{\text {diff }}$ while it decreases for low $L_{\text {diff }}$ (blue arrows in Fig. 3). To explain this phenomenon, we remind that raising the penetration depth increases in any case the number of carriers generated deeper in the bulk. As these carriers have less probability to be collected by the junction, they contribute to increase the injection as long as recombination is low (high $L_{\mathrm{diff}}$ ). If, however, recombination is high, these deeply generated carriers recombine more efficiently in the volume and carrier concentration drastically reduces at low $L_{\text {diff }}$.

This phenomenon tends to attenuate while going to lower $\sigma_{0}$ until a point where injection decreases with increasing penetration depth also for high $L_{\text {diff }}$ (red arrows in Fig. 3). In this case, the generation distribution is so sharp laterally that lateral diffusion dominates the diffusion process leading to the observed sharp reduction of injection. Then, increasing generation depth favors a more efficient lateral diffusion because occurring on a larger extend in $z$ which explains the decrease of carrier maximum density.

The reason why the injection can be several decades lower than the uniform illumination case for small $\sigma_{0}$ is because of the diffusion of carriers generated in the center of the beam occurs in three dimensions instead of one dimension for the uniform illumination case. Having three degrees of freedom, the carriers scatter more efficiently which reduces their accumulation and thus the maximum of injection.

4.4 Parameterization The integrals of Eq. (34) or (37) are unfortunately not analytically solvable and, additionally to their numerical evaluation, an optimization procedure is necessary to locate the maximum of injection in depth. It will be shown later in this section that the number of independent variables in the integral can be reduced from three $\left(\sigma_{0}, L_{\mathrm{diff}}, \alpha\right)$ to two using dimensionless combinations of any of the three variables.

It was discussed in the Section 3 that the maximum injection for uniform illumination can be put as a function of $X=\alpha \cdot L_{\text {diff }}$. Therefore, adopting the same choice here for the first variable allows expressing the present parameterization as a correction to the uniform illumination model.

For a polynomial parameterization, the second variable is then best chosen if it gets to 0 when $\sigma_{0}$ tends to infinity, that is the uniform illumination case. The variable
$Y=\left(\alpha \cdot \sigma_{0}\right)^{-1}$ meets this requirement and additionally allows to determine an upper bound beyond which the parallel beam approximation and so the parameterization is no more valid. The constraint of Eq. (16) on $\sigma_{0}$ leads to a condition on $Y$ described by Eq. (38) and plotted in Fig. 4 for crystalline silicon.

$$
Y<\frac{1}{8} \sqrt{\frac{|\tilde{n}|}{k}}
$$

Making the change of variable $K=k / \alpha$ in Eq. (37) and observing that $p_{00, \text { laser }}=p_{00 \text {,uni }} \cdot(2 X \cdot Y)^{-2}$ if $\Phi_{\text {uni }}=\Phi_{0 \text {, laser }}$ (Eqs. (23) and (35)) leads to

$$
\begin{aligned}
p\left(0, z_{\mathrm{n}}\right)= & p_{00, \text { uni }} \cdot \mathrm{e}^{-\alpha z_{j}}(2 X \cdot Y)^{-2} \int_{0}^{\infty} \frac{K \cdot \exp \left(-K^{2} Y^{-2} / 8\right)}{K^{2}+X^{-2}-1^{2}} \\
& \times\left(\mathrm{e}^{-z_{\mathrm{n}}}-\mathrm{e}^{-\sqrt{K^{2}+X^{-2} z_{\mathrm{n}}}}\right) \mathrm{d} K,
\end{aligned}
$$

using the normalized $z$ variable $z_{\mathrm{n}}=\alpha z$.

Therefore, maximizing the integral with respect to $z_{\mathrm{n}}$ brings a function of $X$ and $Y$ only, as claimed in the beginning of this section. Expressed this way, the prefactor of the uniform illumination and parallel beam model are identical allowing their direct comparison and parameterization.

Figure 5 shows the maximum of Eq. (39) for the usual experimental range where the parallel beam approximation applies. We first observe that uniform illumination is the upper limiting case and that at high $X$ values all curves decay in $X^{-2}$ with, however, a proportionality factor decreasing with $Y$. For low values of $X$, all curves tend to saturate to 1 corresponding to the case where $L_{\mathrm{diff}}$ and $L_{\alpha}$ become smaller than $\sigma_{0}$, and therefore $\sigma_{0}$ is not the limiting factor for injection.

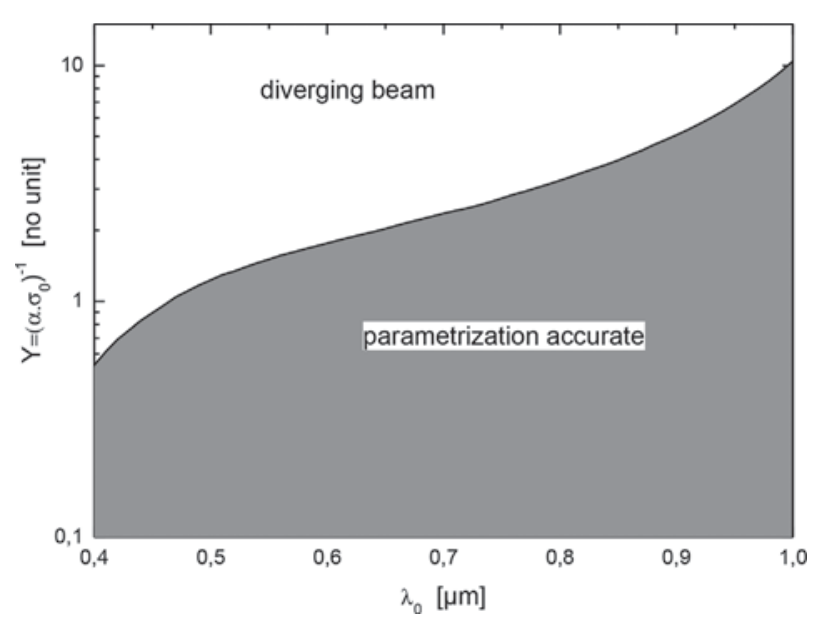

Figure 4 Critical $Y$ value determining for crystalline silicon the range of validity of parameterization of injection as function of the laser wavelength. 


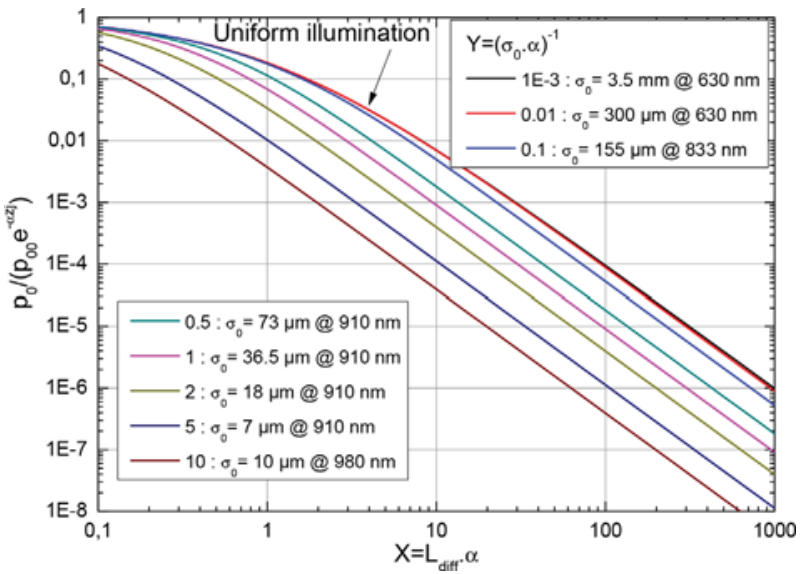

Figure 5 Maximum injection integral in short-circuit condition as a function of $X$ for typical values of $Y$ with reference to typical laser beam wavelength and radius.

The usual experimental range is fully covered by considering $10 \mu \mathrm{m}<L_{\text {diff }}<1 \mathrm{~mm}$ and $1 \mu \mathrm{m}<L_{\alpha}<100 \mu \mathrm{m}$ leading to $0.1<X<1000$. We see from Fig. 4 that the highest allowed value of $Y$ is around 10 (obtained for the highest $\lambda_{0}$ ). On the other hand, a lower bound of $Y=10^{-3}$ can be obtained by considering a very large beam of $\sigma_{0}=1 \mathrm{~mm}$ with the smallest $L_{\alpha}=1 \mu \mathrm{m}$.

The similarity of the shape of all the curves in Fig. 5 suggests that the coefficients $A_{1}$ and $A_{2}$ of the parameterization of the uniform illumination model (Eq. (24)) can be parameterized by polynomial functions of $Y$ such as

$$
A_{1}(Y)=A_{1 \mathrm{u}}\left(1+A_{12} \cdot \mathrm{Y}+A_{13} \cdot Y^{2}+A_{14} \mathrm{Y} /\left(1+A_{15} Y^{2}\right)\right)
$$

$$
A_{2}(Y)=A_{2 \mathrm{u}}\left(1+A_{22} \cdot Y+A_{22} \cdot Y^{2}\right),
$$

in which $A_{12}=0.2985, \quad A_{13}=0.0171, \quad A_{14}=-1.741$, $A_{15}=1.7997, A_{22}=7.8979$, and $A_{13}=1.7997$ using directly the coefficients $A_{1 \mathrm{u}}$ and $A_{2 \mathrm{u}}$ of the uniform illumination parameterization given at the end of the Section 3 .

This parameterization is accurate for most of the regions of interest to $3-4 \%$ as one can see from Fig. 6. There are, however, some domains where the error reaches $10-12 \%$ absolute and where a correction according to Fig. 6 might be recommended to enhance the accuracy.

5 Model for a diverging beam The generation function of the case of a diverging beam is obtained straightforwardly from Eqs. (9) and (10) with $d(z)=1+\left(z / z_{\mathrm{r}}\right)^{2}$ and shifting the $z$-axis by $z_{j}$ (see end of the Section 2.1)

$$
\begin{aligned}
g(r, z)= & \sigma_{0}^{2} g_{0} \frac{\exp \left(-\alpha\left(z+z_{\mathrm{j}}\right)\right)}{\sigma_{0}^{2}+U^{2}\left(z+z_{\mathrm{j}}\right)^{2}} \\
& \times \exp \left(-\frac{2 r^{2}}{\sigma_{0}^{2}+U^{2}\left(z+z_{\mathrm{j}}\right)^{2}}\right),
\end{aligned}
$$

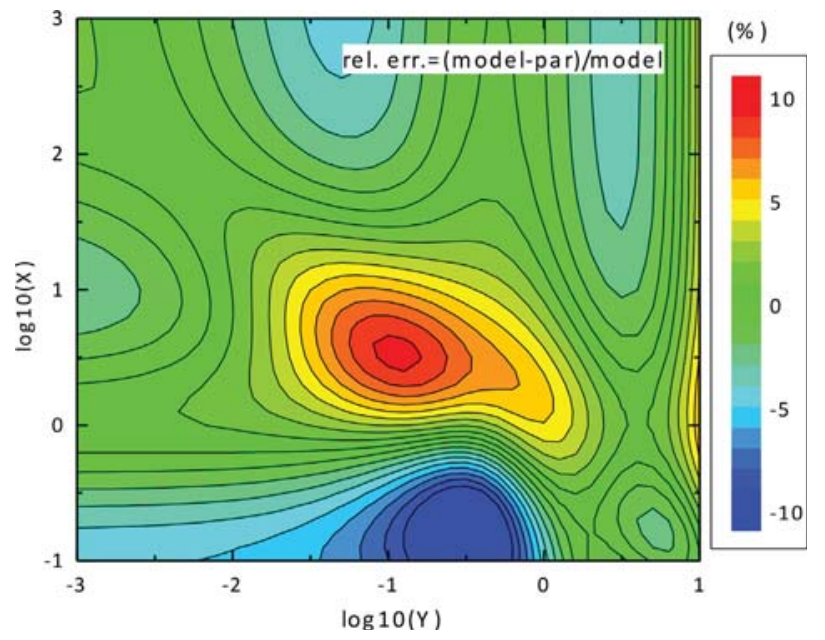

Figure 6 Relative error of the parametrization (Eqs. (24), (40), (41)) for its whole $X, Y$ range of validity.

introducing $U=\sigma_{0} / z_{\mathrm{r}}=\left(\sigma_{0 \min } /|\tilde{n}|\right) / \sigma_{0}$. We see from Eq. (42) that integrating the junction thickness in this generation function does not lead to a constant multiplying factor like for the previous cases and will change the expression of the carrier distribution as shown later.

One can remark that the above form of Eq. (42) is similar to the one used by Marek (Eq. (4) in Ref. [19]) for describing the beam divergence. Marek then implicitly neglected the effect of absorption on the beam curvature which justification is provided in the Section 2 of this article.

The effect of the divergence on the generation function can be best shown in Fig. 7 where the normalized depth distribution of the generation in the center has been represented for various radii but same wavelength.

We see that the curve for $\sigma_{0}=5 \mu \mathrm{m}$ and $10 \mu \mathrm{m}$ are superposed because only the absorption in depth contributes to the generation decay. We then observe that for smaller $\sigma_{0}$ the decay becomes steeper because, additionally to the absorption of the beam, the scattering due to its divergence contributes to the generation decrease.

The thickness of the junction matters therefore even more in this case for determining the injection maximum.

We can remark in Fig. 7 (bottom) that the strong effect of the divergence on the generation of a beam with small $\sigma_{0}$ is less pronounced on the injection compared to a beam with large $\sigma_{0}$. A significant deviation between the two theories is observed only for a very small beam $\left(\sigma_{0}>1 \mu \mathrm{m}\right)$.

We observe that although considering such highly focused beam, the maximum injection is less affected than the deeper part of the injection. The correction for a diverging beam is thus significant but not as much as might has been expected from such a different generation profile.

With Eq. (42) one can rewrite Eq. (5) in Ref. [19] in the following form 


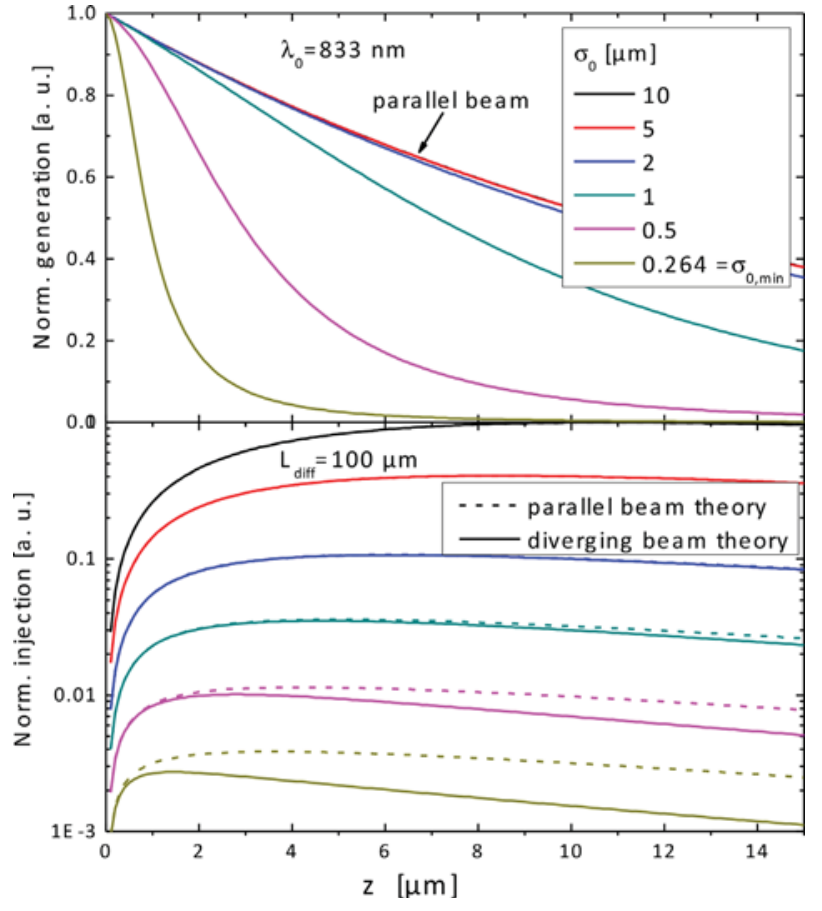

Figure 7 Generation profile in the center of the beam for a laser at $\lambda_{0}=833 \mathrm{~nm}$ and various beam radii (top) and induced normalized injection in the bulk with $L_{\text {diff }}=100 \mu \mathrm{m}$ considering a parallel beam theory or a diverging beam theory (bottom).

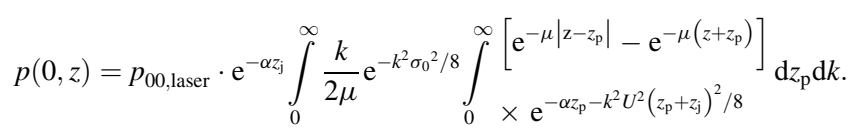

The integral in $z_{\mathrm{p}}$ can be solved analytically leading to

$p(0, z)=p_{00, \text { laser }} \cdot \sqrt{\frac{\pi}{2}} \int_{\times}^{\infty}\left[\begin{array}{l}\exp \left(-\frac{1}{8} k^{2} \sigma_{0}{ }^{2}\right) \frac{1}{U \cdot \mu} \exp (-\mu z) \\ 0\end{array}\left[\begin{array}{l}\exp \left(\Phi_{1}{ }^{2}+\mu z_{j}\right) \\ \exp (2 \mu z) \cdot \operatorname{erfc}\left(\Phi_{1}+\Phi_{3}\right) \\ -\operatorname{erfc}\left(\Phi_{1}+\Phi_{4}\right)\end{array}\right]\right] \cdot \mathrm{d} k$

with

$$
\begin{aligned}
& \Phi_{1}=\sqrt{2}\left(\frac{\alpha+\mu}{k U}\right) ; \quad \Phi_{2}=\sqrt{2}\left(\frac{\alpha-\mu}{k U}\right), \\
& \Phi_{3}=\frac{k U}{2 \sqrt{2}}\left(z+z_{\mathrm{j}}\right) ; \quad \Phi_{4}=\frac{k U}{2 \sqrt{2}} z_{\mathrm{j}} .
\end{aligned}
$$

The evaluation of the $\exp \left(x^{2}\right) \operatorname{erfc}(x)$ like term is performed as described in the appendix of Ref. [25]. The last term of Eq. (44) is also problematic to compute in the sense

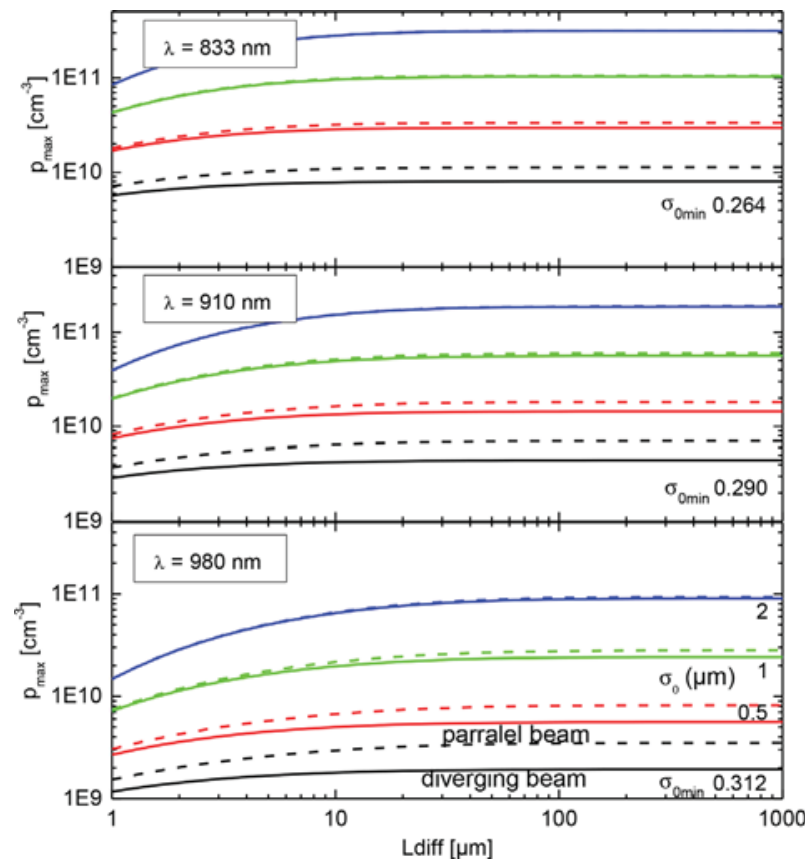

Figure 8 Comparison of the maximum injection induced by laser beams with small radii (given in the bottom graph while the wavelength dependent $\sigma_{0 \min }$ is given in every graph) computed from parallel beam and diverging beam theory in the condition given in Fig. 3.

that $\Phi_{2}+\Phi_{3}$ might become negative. This leads to an $\operatorname{erfc}\left(\Phi_{2}+\Phi_{3}\right)$ values between 1 and 2 that multiplied a large positive exponential which in turn leads to a computational overflow if computed straight forwardly. In such case, using the fact that $\operatorname{erfc}(-x)=2-\operatorname{erfc}(x)$, the 2 cancels out in the difference between the two erfc functions. Therefore, if $\Phi_{2}+\Phi_{3}<0$ one should evaluate $\operatorname{erfc}\left(-\left(\Phi_{2}+\Phi_{4}\right)\right)-$ $\operatorname{erfc}\left(-\left(\Phi_{2}+\Phi_{3}\right)\right)$ instead of $\operatorname{erfc}\left(\Phi_{2}+\Phi_{3}\right)-\operatorname{erfc}\left(\Phi_{2}+\Phi_{4}\right)$.

One can see in Fig. 8 that for such highly focused beam, the difference in injection observed between a parallel beam and a diverging beam theory increases with the wavelength in agreement with the rise of the limit in $\sigma_{0}$ between the two theories (Fig. 1). Indeed for the beam being less absorbed, the divergence of the generation function is more pronounced and with a stronger implication on the injection distribution.

In agreement with the last comment, we observe in Fig. 8 that the discrepancy in injection is, however, reduced to maximum of a factor 2 that has to be compared to 3 to 5 decades that is the discrepancy between the maximum injection induced by uniform illuminations and such focused laser. The beam divergence for extremely focused beam remains therefore a minor correction to the value of the maximum injection that has to be considered only for highly accurate results.

6 Conclusions In various LBIC systems, the accurate knowledge of the maximum injection induced is necessary 
to ensure to stay below a certain threshold (ensuring lowinjection condition in evaluating local defect recombination) or reach a defined value (use of a laser as an illumination bias).

In this context, we demonstrated that the maximum injection of a laser beam is always lower than the one of uniform illumination at equal maximum illumination flux because of lateral carrier diffusion. The influence of lateral diffusion on the maximum injection value is mainly critical if the laser beam radius is smaller than the diffusion length.

We remark that many systems run into this critical situation on the one hand because of the constraint of a high spatial resolution leading to sharply focused laser beams and on the other hand to the good quality of modern semiconductors leading to high diffusion lengths.

With a straightforward calculation of the laser injection based on the uniform illumination model being erroneous in this critical case, we developed a parallel beam model that includes the influence of the beam radius on injection. For a more fundamental understanding, two expressions of the solution are derived each showing different aspects of the model. An accurate parameterization of this model provides a fast correction of the laser power focusing for most common LBIC systems and materials.

In the case of high resolution LBIC systems, the laser beam may be so sharply focused that the divergence of the beam has to be taken into account for an accurate estimation of the injection. A criterion is, therefore, provided to decide whether a parallel beam theory or a diverging beam theory has to be chosen for highly accurate results.

In the case of a diverging beam, several complications are in the way of defining the generation function, such as the non-applicability of the Lambert law, the distortion of the wave curvature due to absorption and the broadening of the laser beam $d(z)$. Though careful and justified approximation, a simplified but very accurate generation function was derived.

This generation function allowed for a diverging beam theory to be developed and compared to the parallel beam theory. There is a significant discrepancy between the two models for highly focused beams which, however, remain a minor correction with respect to the effect of lateral diffusion.

As very sharply focused lasers deliver an injection that can be 3-5 decades lower than the uniform illumination case, the intensity required to reach standard operating conditions could be very large. Therefore, HR LBIC systems without bias light always operate in low injection (which upper limit can be checked with the model), and they are unsuitable for IQE measurements that could be directly compared to standard condition.

Though not being an ideal situation because of the inhomogeneity of the laser generation function, LBIC system designers desiring to work at operating conditions without bias light can use this model to calibrate the maximum injection of their setup.

In the case of solar LBIC for concentrator applications, the prerequisite of spatial homogeneity of injection is in conflict with the need of a high spatial resolution requiring a sharp focusing of the halogen lamp beam. In such case, the use of the present model is even more necessary to ensure the injection to be at the same maximum level as if the illumination was uniform. Care must be taken in such case to the polychromatic nature of the illumination. A careful approximation on this issue can be performed using a method described in Ref. [6].

\section{References}

[1] M. Stemmer and S. Martinuzzi, Inst. Phys. Conf. 135, 239 (1993).

[2] M. Rinio, H. J. Möller, and M. Werner, Diffus. Defect Data 115, 63 (1999).

[3] W. Warta, Sol. Energy Mater. Sol. Cells 72, 389 (2002).

[4] J. Carstensen, G. Popkirov, J. Bahr, and H. Föll, Sol. Energy Mater. Sol. Cells 76, 599 (2003).

[5] R. Gupta and O. Breitenstein, Proc. SPIE 6616, 661600 (2007).

[6] J. Navas, R. Alcántara, C. Fernández-Lorenzo, and J. MartínCalleja, in: Trichromatic High Resolution-LBIC: A System for the Micrometric Characterization of Solar Cells, Solar Cells - Silicon Wafer-Based Technologies, edited by L. A. Kosyachenko (InTech, Rijeka, Croatia, 2011), ISBN: 978953-307-747-5, DOI: $10.5772 / 19542$.

[7] T. Pernau, P. Fath, and E. Bucher, in: Proc. 29th IEEE Photovoltaic Specialist Conference, pp. 442-445 (2002).

[8] L. A. Bitzer, M. Meseth, N. Benson, and R. Schmechel, Rev. Sci. Instrum. 84, 023707 (2013).

[9] F. J. Vorster and E. E. van Dyk, Rev. Sci. Instrum. 78, 013904 (2007).

[10] A. Kaminski, O. Breitenstein, J. P. Boyeaux, P. Rakotoniaina, and A. Laugier, J. Phys.: Condens. Matter 16, 66991 (2004).

[11] R. Woehl, M. Hörteis, and S. W. Glunz, in: Proc. 23rd EUPVSEC Valencia, Spain, p. 1377 (2008).

[12] J. Martın, C. Fernandez-Lorenzo, J. A. Poce-Fatou, and R. Alcantara, Prog. Photovolt.: Res. Appl. 12, 283 (2004).

[13] R. Corkish, T. Puzzer, and A. B. Sproul, J. Appl. Phys. 84, 5473 (1998).

[14] Sentaurus Device Electromagnetic Wave Solver User Guide, version H-2013.03, Synopsys, p. 69 (2013).

[15] B. E. A. Saleh and M. C. Teich, Fundamentals of Photonics (John Wiley \& Sons, Chichester, UK, 1991), chap. 5.

[16] S. R. Seshadri, Opt. Lett. 29, 1179 (2004).

[17] K. Thyagarajan and A. Ghatak, Lasers-Fundamentals and Applications, 2nd ed. (Springer, New York, Dordrecht, Heidelberg, London, 2010).

[18] C. C. Davis, Lasers and Electro-Optics (Cambridge University Press, New York, 2004).

[19] J. Marek, J. Appl. Phys. 55, 318 (1984).

[20] C. Donolato, Phys. Status Solidi A 65(649), (1981). 
[21] B. Davies, Integral Transforms and Their Applications (Texts in Applied Mathematics), 3rd ed., Vol. 41 (Springer, New York, 2002).

[22] M. A. Stegun and I. A. Abramowitz, Handbook of Mathematical Functions (Dover, New York, 1972)

[23] G. Micard, Ph.D. thesis, University of Konstanz (2011).
[24] I. S. Gradshteyn and I. M. Ryzhik, Table of Integrals, Series, and Products, 7th ed. (Elsevier, Academic Press, London, Munich, Amsterdam, 2007).

[25] G. Micard, G. Hahn, A. Zuschlag, S. Seren, and B. Terheiden, J. Appl. Phys. 108, 034516 (2010). 